\title{
Fingerprint-based Location Estimation with Virtual Access Points
}

\author{
A.K.M. Mahtab Hossain, Hien Nguyen Van, Wee-Seng Soh \\ Department of Electrical \& Computer Engineering \\ National University of Singapore, Singapore \\ Email: $\{g 0500774$, elenvh, elesohws $\} @$ nus.edu.sg
}

\begin{abstract}
Location fingerprinting techniques generally make use of existing wireless network infrastructure. Consequently, the positions of the access points (APs), which constitute an integral part of a location system, will invariably be dictated by the network administrator's convenience regarding data communication. But the localization accuracy of fingerprint-based solutions is largely dependent on the APs' placements over the area. In this paper, we developed the idea of Virtual Access Point (VAP), where one can have AP's functionality at a desired position for localization purpose, without physically placing an AP there. We argue that, placing VAPs at favorable positions helps to improve localization accuracy. VAP also serves the purpose of virtually increasing the number of APs over the localization area, which according to previous works should enhance the localization accuracy further. We test the feasibility of our VAP idea both analytically and experimentally. Finally, we present our results using a well-known localization algorithm, namely, K-Nearest Neighbor, when our VAP idea is implemented. The findings are quite encouraging, which report significant improvement in the localization accuracy.
\end{abstract}

Keywords: Positioning Systems, Indoor Localization, Virtual Access Point, Location Fingerprint, Bluetooth.

\section{INTRODUCTION}

Traditional location estimation solutions specifically designed for indoors generally require dedicated infrastructure based on infrared, radio frequency, or ultrasound technologies solely for positioning purpose [1]-[3]. Recently, there has been a growing interest in indoor localization techniques that rely on in-building communications infrastructure (e.g., Wi$\mathrm{Fi}$, Bluetooth, etc.) mainly because it allows the design of an easily deployable low-cost positioning system. Most of these approaches utilize location fingerprinting techniques [4]-[6], which provide reasonable positioning accuracy but entail a laborious training phase in order to construct the radio-map. Fingerprinting based systems generally have two phases - offline training phase and online location estimation phase. During the offline phase, the location fingerprints (i.e., signal strength samples) at the selected locations of interest are collected, yielding the so-called radio map. In order to differentiate between various locations, the entire area is usually covered by a rectangular grid of points. During the online location determination phase, the signal strength samples received at the access points (APs) from the mobile node $(\mathrm{MN})$, or vice versa, will be sent to a central server. The server then uses some algorithm to estimate the MN's position, and reports it back to the MN (or the application requesting the location information).

Some prior works [6], [7] have suggested that the accuracy of a fingerprint-based location system is somewhat dependent on the APs' placements. But the main idea behind these inexpensive fingerprint-based techniques is that, such schemes provide location information as value-added services on top of the existing infrastructure for data networks (e.g., WLANs). As a result, the APs' positions would largely be dictated by the ease of data communication. Moreover, for a fingerprintbased location system, it is not clear how many APs need to be "heard" at a given point for a particular accuracy and precision ${ }^{1}$. The general consensus is that, the higher the number of APs, the better the performance reported - some works [6] have also validated this claim with both analytical and experimental findings. Therefore, a positioning system administrator may benefit from as many APs as possibly could be "heard" at a particular position, in order to estimate that location more accurately.

In this paper, we have developed the idea of virtual access point (VAP), which tries to achieve the aforementioned requirements regarding a positioning system. We contend that there are certain relationships among the APs' received signal strengths from a MN. Utilizing this notion, we can generate VAPs at desired positions that would aid localization, thereby eliminating the limitations imposed by fixed AP placements that were possibly installed with facilitating data communication in mind. Moreover, we argue that, by selecting good positions for VAPs' placement, the localization accuracy would see a monotonic increase with the number of VAPs, at no additional hardware cost. In short, we contend that, our cost-effective VAP-based positioning system could improve the localization accuracy offered by current fingerprint-based techniques.

The rest of the paper is organized as follows. In Section II, we elaborately discuss our virtual access point based system from both analytical and experimental point of view. In Section III, we present the localization experimental results incorporating our VAP idea. Finally, we present in Section IV the conclusions drawn, as well as our future work.

\footnotetext{
${ }^{1}$ Location accuracy is the error distance deviated from the actual position, while precision is the percentage of measurements that fall within that distance of accuracy.
} 


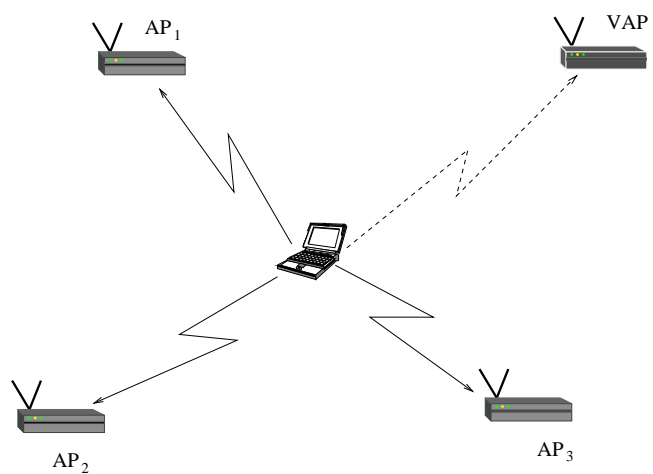

Fig. 1. Creation of VAP from other real APs' signals.

\section{The Concept of Virtual Access Points}

\section{A. Motivation}

In a location system that is aided by VAPs, the received signal strength (RSS) values at such VAPs are estimated from real APs' RSS values. These estimated RSS values are then used together with the real APs' RSS values during the location estimation of a MN, as depicted in Fig. 1. Our VAPbased location system is inspired by two key observations. The first key observation is that, the fingerprint-based location system's designer normally would have to provide location services as a value-added service on top of an existing infrastructure, thereby, having no control over the real APs' placements. However, previous work in the literature, as well as our own experimental results, have observed that certain positions are more favorable than others for the placement of APs regarding localization. Although it would aid localization if additional APs were installed at such locations, it may not always be practical to do so. The second key observation is that, a system's location accuracy is usually enhanced by an increase in the number of APs, as suggested by previous work in the literature. Our VAP-based location system is able to accomplish the advantages suggested by the above two observations, as follows:

- It creates APs (virtual ones) at preferred positions with no additional hardware cost incurred upon the system.

- During the online location determination phase, the number of APs' RSS values are now augmented by the VAPs' RSS values, thereby enhancing the localization accuracy.

\section{B. System Model}

Just like any fingerprint-based location system, our VAPbased system also has the same two phases, namely, the offline training phase and the online location estimation phase. There are only small additional changes in the two phases, which do not incur any substantial burden upon the system. Moreover, it could actually help the system in achieving particular accuracy and precision without extra cost (e.g., without deployment of additional real APs). We now discuss the two phases in detail.

1) Offline Training Phase:

- Collecting Samples: First, we collect RSS samples at the APs from the MN while placing the latter at different
TABLE I

CORRElation MatriX OF OUR TESTBED's ThreE REAL APS' AND TwO MONitoring DEVICES' RSS VAlues

$\begin{array}{cccccc} & \mathrm{AP}_{1} & \mathrm{AP}_{2} & \mathrm{AP}_{3} & \mathrm{VAP}_{1} & \mathrm{VAP}_{2} \\ \mathrm{AP}_{1} & 1.0000 & -0.2190 & 0.1457 & -0.2333 & 0.4898 \\ \mathrm{AP}_{2} & -0.2190 & 1.0000 & 0.0177 & -0.0432 & -0.0875 \\ \mathrm{AP}_{3} & 0.1457 & 0.0177 & 1.0000 & -0.7785 & 0.1348 \\ \mathrm{VAP}_{1} & -0.2333 & -0.0432 & -0.7785 & 1.0000 & -0.3278 \\ \mathrm{VAP}_{2} & 0.4898 & -0.0875 & 0.1348 & -0.3278 & 1.0000\end{array}$

locations over our testbed, similar to the traditional fingerprint-based systems. The only difference is that, RSS samples are also collected by placing monitoring devices at the potential positions of VAPs at the same time. Now, if there are $N$ APs, and that $M$ VAPs are to be generated, each RSS sample vector in the database takes the form of a $(N+M)$-dimensional vector:

$\left[P\left(\mathrm{AP}_{1}\right), P\left(\mathrm{AP}_{2}\right) \ldots P\left(\mathrm{AP}_{N}\right), P\left(\mathrm{VAP}_{1}\right), \ldots P\left(\mathrm{VAP}_{M}\right)\right]$, where $P\left(\mathrm{AP}_{i}\right)$ is the RSS at the $i^{\text {th }} \mathrm{AP}$, and $P\left(\mathrm{VAP}_{i}\right)$ is the RSS at the $i^{\text {th }}$ VAP position. Without loss of generality, we assume that the monitoring devices' hardware are of the same type as those of the APs.

- Statistical Analysis and Creation of VAP Model: Various statistical tools can be applied to the gathered training samples in order to create an appropriate VAP model. For example, in Table I, we have shown the space correlations of our testbed's three APs' RSSs and two monitoring devices' RSSs from a MN, when the two monitoring devices are placed at the VAPs' positions indicated in Fig. 5. The absolute value of each entry in Table I indicates the degree of linear dependence between a pair of APs with regard to the RSS samples collected during the training phase. The strong correlation between $\mathrm{AP}_{3}$ 's RSS and the first monitoring device's RSS (placed at VAP $_{1}$ 's position in Fig. 5) made us believe that, there are certain relationships between two APs' RSSs, such that knowing one or more APs' RSSs may give us a measure of another AP's RSS. Hence, the idea of VAP has blossomed. We have used simple least-mean square multivariate linear regression model in order to forecast a VAP's RSS:

$$
P\left(\operatorname{VAP}_{j}\right)=a_{j_{0}}+\sum_{i=1}^{N} a_{j_{i}} P\left(\mathrm{AP}_{i}\right) .
$$

Here, $P\left(\mathrm{AP}_{i}\right)$ and $P\left(\mathrm{VAP}_{j}\right)$ represent the RSSs of the $i^{\text {th }} \mathrm{AP}$ and the $j^{\text {th }}$ VAP from the MN, respectively. Also, the $a_{j_{i}}$ 's are the regression coefficients with regard to the $j^{\text {th }}$ VAP, and that $N$ real APs are considered in inferring this particular VAP's RSS equation. The calculation of regression coefficients is shown in Appendix A. Using 


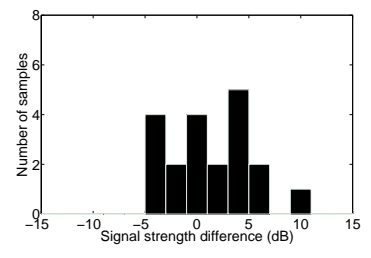

(a) VAP Position 1

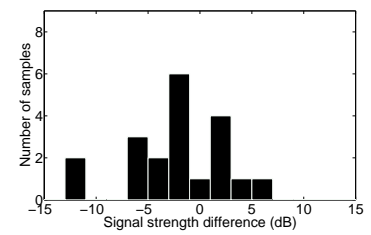

(c) VAP Position 3

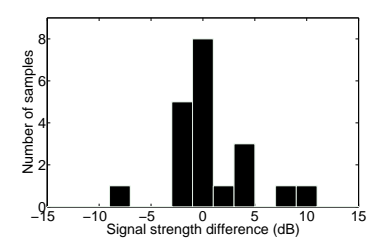

(b) VAP Position 2

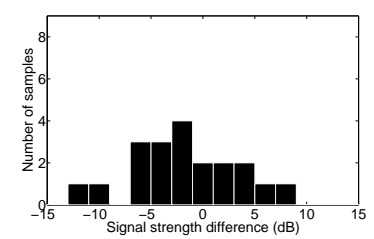

(d) VAP Position 4
Fig. 2. Histograms of signal strength differences between the actual signals and the signals predicted using linear regression, for 4 different VAP positions.

the shadowing model under some simplifying assumptions, we have also validated that our linear regression approximation is a reasonable one, which we will discuss in Section II-C.

Fig. 2 illustrates our linear regression model's performance when the VAP is placed at four different locations. For this experiment, we have used one set of training samples to generate the RSS prediction formula for the VAP, and tested its performance on another set. In accordance with our previous discussion, we see that, placing VAPs at different locations does not provide the same performance in predicting RSS. In other words, the prediction error is small at some VAP positions (e.g., Fig. 2(b)), whereas at other VAP positions it may be a bit high (e.g., Fig. 2(a)).

Before starting with the sample collections, it is helpful to perform simulations to obtain some intuition about where to place the monitoring devices, which will ultimately be the positions of the VAPs. In our case, simulations using the simplified shadowing model (without the variation factor) gave us insightful pointers in placing the VAPs (see Fig. 3). We have empirically obtained the path-loss exponent $\beta$ of the shadowing model from the collected training samples, in order to model our testbed in the simulations. To obtain the results of Fig. 3(a) and 3(b), we have fixed the APs but varied the positions of the VAPs. We then generated artificial RSS samples over the whole localization area according to the simplified shadowing model for each position of the VAP, and calculated its RSSs' correlation with respect to the fixed AP shown in the figures. In both figures, we see that, those VAP positions that are close to the fixed APs tend to have high correlation factors. An interesting observation is that, from Fig 3(b), the VAP positions at the opposite end of $\mathrm{AP}_{3}$ also have better prospects, as can be seen from the strongly negative correlation factors. The position of our $\mathrm{VAP}_{1}$ is eventually chosen to be at the opposite side of $\mathrm{AP}_{3}$, after performing extensive simulations among all

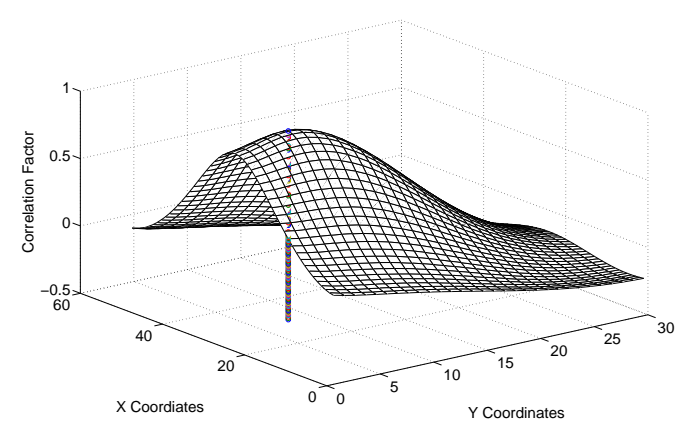

(a) $\mathrm{AP}_{1}$ 's position is fixed - VAP's positions are changed over the whole area giving different correlation factors w.r.t. $\mathrm{AP}_{1}$.

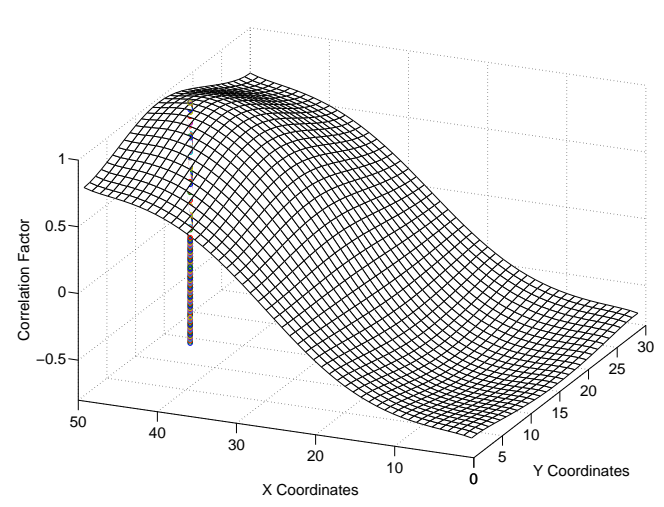

(b) $\mathrm{AP}_{3}$ 's position is fixed - VAP's positions are changed over the whole area giving different correlation factors w.r.t. $\mathrm{AP}_{3}$.

Fig. 3. Using simulations to identify potential VAP positions by plotting the correlation factors of different VAP positions w.r.t. a fixed AP. The AP against which the correlations are calculated is marked with a pillar.

the APs. As will be seen later, our experimental results in Section III-B are also more favorable when we consider $\mathrm{VAP}_{1}$, rather than $\mathrm{VAP}_{2}$ (their positions are indicated by shaded circles in Fig. 5). This strengthens the fact that, simulations can actually help in finding a potential good position for a VAP. In the worst case, one may need to perform the sample collection phase again if the initial choices of VAPs' positions turn out to be bad ones.

2) Online Location Determination Phase: In this phase, only the real APs will be collecting RSS samples from the MN. For example, in Fig. 1, there will be no monitoring device at the VAP's position. Hence, the RSS vector will now have a dimension of $N$, i.e., $\left[P\left(\mathrm{AP}_{1}\right), P\left(\mathrm{AP}_{2}\right) \ldots P\left(\mathrm{AP}_{N}\right)\right]$. By plugging these values into the RSS prediction formulas of each VAP (as in (1)), we will obtain the $M$ VAPs' RSSs. The next steps are similar to any fingerprint-based location system. The only difference is that, instead of using an RSS sample vector of dimension $N$, we are using an RSS vector of dimension $N+M$, in order to infer the location estimate.

\section{Validation of Linear Regression Approximation}

Suppose $P(d)$ and $P\left(d_{r}\right)$ denote the received power of a device at an arbitrary distance $d$ and a close-in reference distance $d_{r}$ from a transmitter, respectively. From the log- 


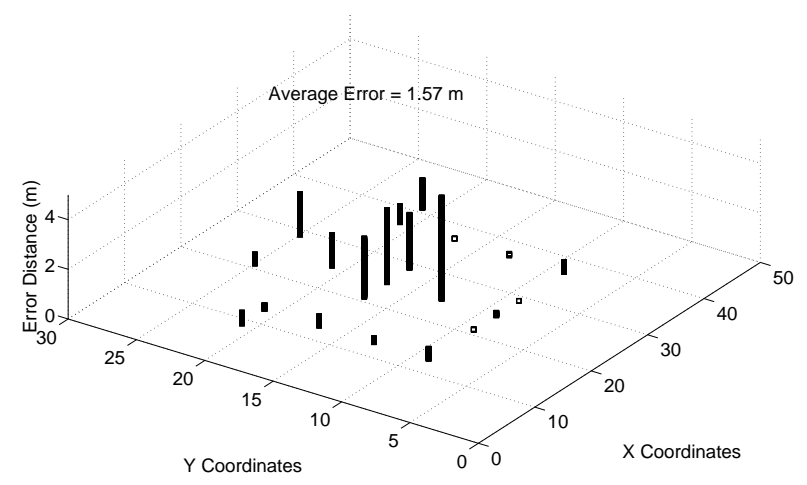

Fig. 4. The distribution of differences between real and predicted distances of a VAP from 20 training points - the error bars are drawn at the training points for which the distances from the VAP were calculated.

normal shadowing model, we get

$$
\left[\frac{P(d)}{P\left(d_{r}\right)}\right]_{\mathrm{dB}}=-10 \beta \log \left(\frac{d}{d_{r}}\right)+X .
$$

The first part of (2) defines the path loss component ( $\beta$ is the path loss exponent) and the second part reflects the variation of the received power at a certain distance $\left(X \sim N\left(0, \sigma_{\mathrm{dB}}\right)\right)$. Eqn. (2) can be rewritten as,

$$
\begin{array}{r}
\left.P(d)\right|_{\mathrm{dBm}}-\left.P\left(d_{r}\right)\right|_{\mathrm{dBm}}-10 \beta \log \left(d_{r}\right)= \\
-10 \beta \log (d)+X .
\end{array}
$$

From (1), we know that,

$$
P(\mathrm{VAP})=a_{0}+\sum_{i=1}^{N} a_{i} P\left(\mathrm{AP}_{i}\right)
$$

Here, $P\left(\mathrm{AP}_{i}\right)$ and $P(\mathrm{VAP})$ represent the RSSs of the $i^{\text {th }} \mathrm{AP}$ and the VAP due to the MN, respectively. Since we assume that the path-loss exponent $\beta$ is the same over our testbed, and that the monitoring device placed at the potential VAP's position collecting RSS samples would be of similar hardware as the real APs, the $\left.P\left(d_{r}\right)\right|_{\mathrm{dBm}}$ and $-10 \beta \log \left(d_{r}\right)$ factors of (3) are similar for all devices. Using this property, (4) can be simplified as

$$
\begin{array}{r}
-10 \beta \log \left(d_{\mathrm{VAP}}\right)+X_{\mathrm{VAP}}= \\
a_{0}-10 \beta \sum_{i=1}^{N} a_{i} \log \left(d_{i}\right)+\sum_{i=1}^{N} a_{i} X_{i},
\end{array}
$$

where $d_{i}$ is the distance between $\mathrm{AP}_{i}$ and the $\mathrm{MN}$, and $X_{i}$ is the variation factor associated with $\mathrm{AP}_{i}$. We assume that all $X_{i}$ 's are independent, so $\sum_{i=1}^{N} a_{i} X_{i}$ follows a normal distribution. For simplicity, by eliminating the Gaussian variation factors from both sides of (5), and assuming that $a_{0}=-10 \beta \log \left(d_{0}\right)$, we get

$$
-10 \beta \log \left(d_{\mathrm{VAP}}\right) \approx-10 \beta \log \left(d_{0}\right)-10 \beta \sum_{i=1}^{N} a_{i} \log \left(d_{i}\right) .
$$

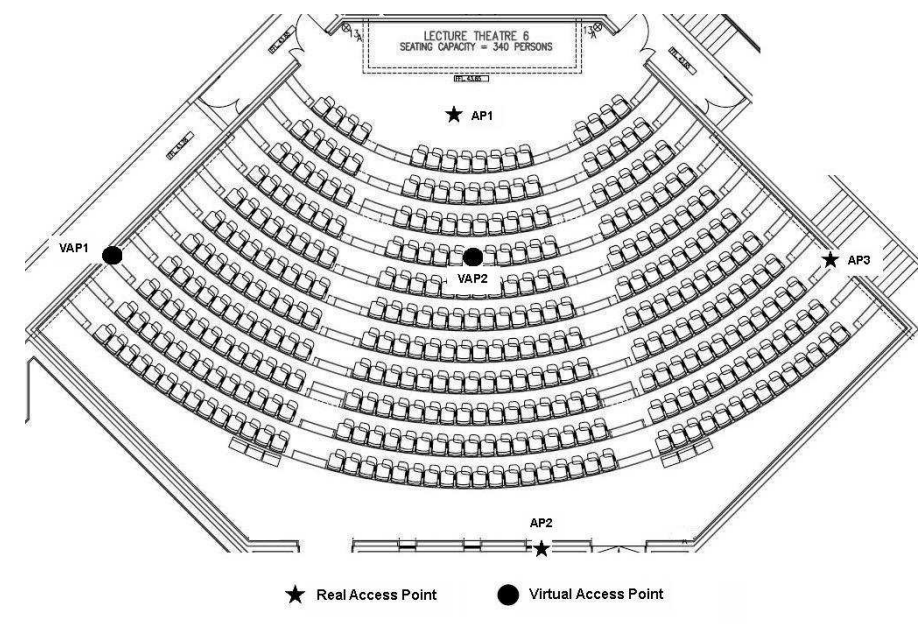

Fig. 5. Our experimental testbed - the three APs are marked as stars, while the two VAPs' positions are shown as shaded circles.

Simplifying (6), we finally obtain

$$
d_{\mathrm{VAP}} \approx d_{0}{ }^{1} d_{1}^{a_{1}} d_{2}{ }^{a_{2}} \ldots d_{N}{ }^{a_{N}} .
$$

Eqn. (7) reflects that the distance of VAP from any training point can be represented using the distances of the APs from the same training point, together with the regression coefficients of the VAP's RSS equation. Fig. 4 shows the differences between real and predicted distances (obtained using (7)) of a VAP from the locations where training samples have been collected. We see that the predictions using (7) are quite good with an average error of only $1.57 \mathrm{~m}$. This validates that our claim of linear regression fit for VAP's RSS is indeed a reasonable one. In order to obtain Fig. 4, we first came up with the regression equation from the training samples, and also calculated the value of $\beta$ empirically to get $d_{0}$. Since we know the distances of the APs from each training point, we just predicted $d_{\text {VAP }}$ using (7) for each case, and compared it with the real distance.

\section{LOCALIZATION EXPERIMENTAL RESULTS}

First, we briefly describe our system's experimental testbed and our data collection procedure. We then present our experimental results. Note that all the experimental results presented in the previous section are also based on this same testbed.

\section{A. Experimental Testbed}

Our experimental testbed is located inside an amphitheater of our school which spans over an area of $540 \mathrm{~m}^{2}$. A $50 \times 30$ grid has been considered to map our whole area. We have used three Aopen MP945 Mini PCs to serve as our APs, which are placed near the ceilings. The locations of these APs are shown in Fig. 5, marked as stars. Each Mini PC is incorporated with a BT-2100 Class 1 Bluetooth adapter, and is also connected to our school's intranet for communicating with the server by means of a wired LAN connection. All our Mini PCs run SuSe 10.1 Linux distribution with the latest BlueZ protocol stack. We have either used the Mini PC or a laptop equipped with the same BT-2100 Bluetooth adapter as the monitoring device 


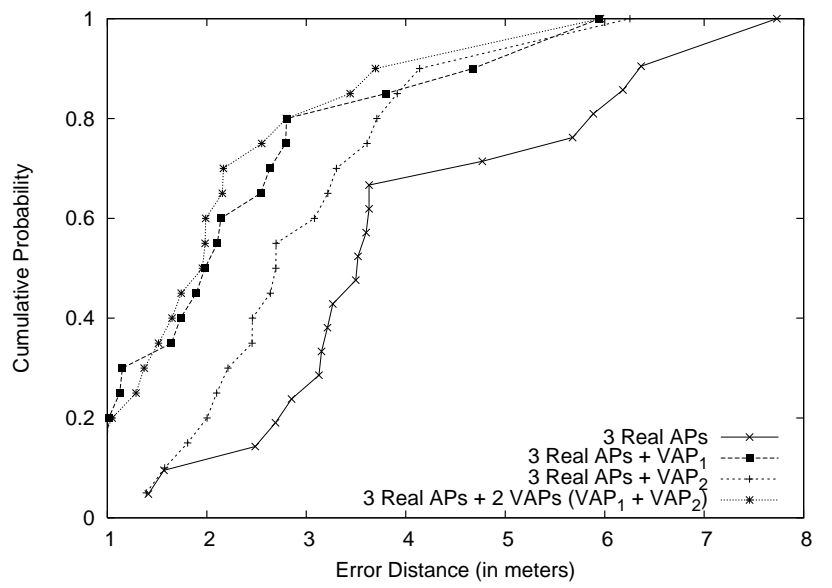

Fig. 6. Performance of various combinations of APs and VAPs for location estimation - the KNN algorithm has been used.

at the potential position of a VAP. Two such VAP positions which we have considered in our experiments are shown in Fig. 5, marked as shaded circles.

\section{B. Data Collection Procedure}

Our Bluetooth APs continuously issue inquiries which the mobile devices respond to. These response packets' information is immediately transferred to our central server, which filter out all the other packets' information apart from the training samples. In other words, only training samples' information will be stored in the database. The central server is also responsible for calculating the location estimate. Note that our Bluetooth adapters provide absolute RSS metric. In addition, rather than using pure RSS values, we have utilized Signal Strength Differences (SSD) [8] to denote a location's fingerprint, which give better performance.

\section{Experimental Results and Findings}

1) Impact of Positions of VAPs: In Section II-B, our simulation results have suggested that $\mathrm{VAP}_{1}$ 's position is a good one. Fig. 6 strengthens this claim, since the localization accuracy achieved when $\mathrm{VAP}_{1}$ is included turns out to be better than the case when $\mathrm{VAP}_{2}$ is included. As we have discussed, not all positions may be favorable for the placements of VAPs. For example, in Fig. 7, the average localization error actually worsens when a VAP is considered at $\mathrm{AP}_{2}$ 's position, compared with a system that does not incorporate any VAP (the leftmost pair of the bars). By inspecting Table I, we observe that $\mathrm{AP}_{2}$ 's $\mathrm{RSSs}$ have very little correlation with the other APs' signals, which may have ultimately resulted in a bad prediction model. On the contrary, the inclusion of $\mathrm{VAP}_{1}$ reduces the average localization error immensely, as evident in Fig. 7 (the rightmost pair of the bars). Upon inspecting Table I, we see that $\mathrm{VAP}_{1}$ has the best correlation factors w.r.t. other APs, which helps explain why our prediction model utilizing the other APs' RSSs to predict the RSS at $\mathrm{VAP}_{1}$ has turned out well. Our simulation results previously presented in Section II-B have also foretold that the position of $\mathrm{VAP}_{1}$ is a good one.

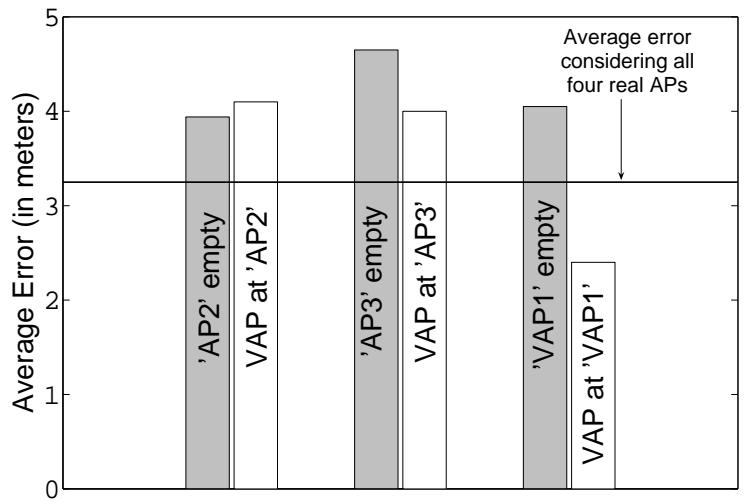

Fig. 7. Average localization errors when various combinations of APs are considered - for this experiment, we have considered a maximum of four real APs (placed at the positions labeled as ' $\mathrm{AP}_{1}$ ', ' $\mathrm{AP}_{2}$ ', ' $\mathrm{AP}_{3}$ ' and ' $\mathrm{VAP}_{1}$ ', in Fig. 5). In each case of the three pairs of bars, the right bar within the pair shows the result when a VAP has been considered in place of one of the four APs. The left bar within the pair shows the result when neither a VAP nor an AP has been considered at that same place (i.e., only left with three real APs). The horizontal line gives the error when all four real APs are considered.

It is interesting to note in Fig. 7 that, the use of $\mathrm{VAP}_{1}$ (refer to the rightmost bars) actually performs better than the case where a real AP is placed at $\mathrm{VAP}_{1}$ 's position (refer to the horizontal average error line at around $3.3 \mathrm{~m}$ ). This could be due to two reasons - firstly, that position was the best in terms of placing a VAP, and secondly, the collected samples incorporated much noises for the real AP's signals when the AP was placed there.

2) Impact of the Number of VAPs: From Fig. 6, it is evident that the inclusion of a VAP at a reasonable position improves the localization accuracy compared to the scenario when there is none. Moreover, we also find that, a location system with two VAPs outsmarts those systems with only one VAP. This phenomenon complies with the findings of prior works that report monotonic increase in accuracy with the number of APs. Our contribution is that, we can achieve it without actually employing additional APs in that area; we only need to create VAPs at favorable positions from the already existing APs.

\section{CONCLUSION AND FUture WORK}

In this paper, we have proposed a new VAP-based positioning system, which aims to improve the localization accuracy and cost-effectiveness of a system that utilizes existing infrastructure to provide location services. We have discussed intuitive guidelines about how to choose the VAPs' positions, and found that our linear regression based prediction formula for VAP's RSS has performed quite well. Based on our analysis and experimental results, the following conclusions can be drawn:

- Our main contribution is that, for a system with limited number of APs, the localization accuracy may be improved significantly with VAPs. We also contend that the choice of the VAPs' positions plays an important role in achieving that goal, and hence it should be done carefully.

- Fingerprint-based positioning system is known to be costeffective itself, because of the use of existing infrastruc- 
ture. VAP can further reduce the cost, since it provides AP's functionality for localization purpose, without even being a physical entity.

- The mismatch of dimension of RSS vectors collected during offline and online phases is a known problem for any fingerprint-based positioning system, because of the absence of some APs in the online phase. VAP may help in that regard by deriving all the APs' RSS prediction formulas based on the training samples. When one of them is off, its RSS value can be predicted using the other live APs' signals.

- At first glance, VAP-based positioning system may give the impression of putting substantial burden on the training phase of a fingerprint-based location system. But we have seen that, if the initial choices for placing the VAPs are promising, it does not incur any additional burden for the training phase.

- The advantages offered by our VAP-based positioning system can be realized with any choice of existing fingerprint-based localization algorithms. In this paper, we have only used the well-known KNN for all the localization experiments performed.

In summary, our VAP-based positioning system improves the localization accuracy offered by current fingerprint-based techniques with no additional hardware cost. In the following, we list some future directions that we foresee:

- Only linear regression has been used to predict VAP's RSS from the other APs' signals - more complex relationships may provide better results.

- In our testbed, the number of APs were low, so we have used all of them to obtain a VAP's model. Consider a scenario where many APs are available. Based on the nature of those APs' signals, only a few of them may be used for a particular VAP's model. In other words, preprocessing of the signals from the APs may lead us to select a subset of the available APs, rather than using all, and this might provide interesting results.

- Our current VAP-based positioning system is based on Bluetooth wireless technology, but the idea is generic enough to be used with any other technology. It would also be challenging to apply our VAP idea where multiple wireless technologies' APs coexist, and to incorporate those diverse set of APs' signals to predict a VAP model.

\section{ACKNOWLEDGMENTS}

The research reported in this paper was supported by the Ministry of Education of Singapore, AcRF Tier 1 funding, under Grant No. R-263-000-320-112 and R-263-000-320-133.

\section{APPENDIX A}

\section{CALCUlation of RegRession COEFFicients of VAP}

We calculate the coefficients of our VAP's linear regression model in (4), subject to the constraint $\sum_{i=0}^{N} a_{i}=1$. Let us define the deviation of VAP's predicted RSS from the real one with regard to the $l^{\text {th }}$ training location as $\Delta \varepsilon_{l}=$ $\left[\begin{array}{llllll}a_{0} & a_{1} & \ldots & a_{N}-1\end{array}\right]\left[\begin{array}{llllll}1 & P_{l}^{1} & P_{l}^{2} & \ldots & P_{l}^{N} & P_{l}^{\mathrm{VAP}}\end{array}\right]^{T}=\mathcal{A}^{T} \mathcal{P}_{l}$, where
$\mathcal{A}=\left[\begin{array}{lllll}a_{0} & a_{1} & \ldots & a_{N} & -1\end{array}\right]^{T}, \mathcal{P}_{l}=\left[\begin{array}{llllll}1 & P_{l}^{1} & P_{l}^{2} & \ldots & P_{l}^{N} & P_{l}^{\mathrm{VAP}}\end{array}\right]^{T}$. Here, $P_{l}^{j}$ and $P_{l}{ }^{\mathrm{VAP}}$ represent the RSSs of the $j^{\text {th }}$ AP and the monitoring device placed at the VAP position, respectively, when the $\mathrm{MN}$ is stationed at training location $l$. Now, our aim is to minimize $\left(\Delta \varepsilon_{l}\right)^{2}$ over all the $L$ training locations. Consequently, the optimization problem becomes,

$$
\text { Minimize } \begin{aligned}
\sum_{l=l}^{L}\left(\Delta \varepsilon_{l}\right)^{2} & =\sum_{l=1}^{L}\left(\mathcal{A}^{T} \mathcal{P}_{l}\right)\left(\mathcal{A}^{T} \mathcal{P}_{l}\right)^{T} \\
& =\sum_{l=1}^{L}\left(\mathcal{A}^{T} \mathcal{P}_{l}\right)\left(\mathcal{P}_{l}^{T} \mathcal{A}\right) \\
& =\mathcal{A}^{T} \sum_{l=1}^{L}\left(\mathcal{P}_{l} \mathcal{P}_{l}^{T}\right) \mathcal{A}=\mathcal{A}^{T} \mathcal{B} \mathcal{A}
\end{aligned}
$$

subject to $\mathcal{C}^{T} \mathcal{A}=1$, where $\mathcal{C}=\left[\begin{array}{lllll}1 & 1 & \ldots & 1 & 0\end{array}\right]^{T}$ and $\mathcal{B}=$ $\sum_{l=1}^{L}\left(\mathcal{P}_{l} \mathcal{P}_{l}^{T}\right)$. The objective function incorporating the constraint can be written as,

$$
\mathcal{L}(\mathcal{A}, \lambda)=\mathcal{A}^{T} \mathcal{B} \mathcal{A}+\lambda\left(1-\mathcal{C}^{T} \mathcal{A}\right)
$$

where $\lambda$ is Lagrange multiplier. Subsequently, we can come up with the following equations,

$$
\begin{aligned}
\frac{\partial \mathcal{L}}{\partial \mathcal{A}}\left(\mathcal{A}^{*}, \lambda^{*}\right) & =2 \mathcal{B} \mathcal{A}^{*}-\lambda^{*} \mathcal{C}=0 \\
& \Rightarrow \mathcal{A}^{*}=\frac{\lambda^{*} \mathcal{B}^{-1} \mathcal{C}}{2} \\
\frac{\partial \mathcal{L}}{\partial \lambda}\left(\mathcal{A}^{*}, \lambda^{*}\right)= & 1-\mathcal{C}^{T} \mathcal{A}^{*}=0 \\
\Rightarrow \quad & 1-\frac{\lambda^{*} \mathcal{C}^{T} \mathcal{B}^{-1} \mathcal{C}}{2}=0, \quad \text { using (8) } \\
\Rightarrow \quad \lambda^{*} & =\frac{2}{\mathcal{C}^{T} \mathcal{B}^{-1} \mathcal{C}}
\end{aligned}
$$

Plugging in the value of $\lambda^{*}$ from (9) into (8), we obtain the optimum values of the regression coefficients, $\mathcal{A}^{*}=\frac{\mathcal{B}^{-1} \mathcal{C}}{\mathcal{C}^{T} \mathcal{B}^{-1} \mathcal{C}}$.

\section{REFERENCES}

[1] R. Want, A. Hopper, V. Falcão, and J. Gibbons, "The active badge location system," ACM Trans. on Information Systems, vol. 10, no. 1, pp. 91-102, Jan. 1992.

[2] A. Ward, A. Jones, and A. Hopper, "A new location technique for the active office," IEEE Personal Communications, vol. 4, no. 5, pp. 42-47, Oct. 1997.

[3] N. Priyantha, A. Chakraborty, and H. Balakrishnan, "The cricket locationsupport system," in Proc. MobiCom, Aug. 2000, pp. 32-43.

[4] P. Bahl and V. N. Padmanabhan, "RADAR: An in-building RF-based user location and tracking system," in Proc. IEEE INFOCOM, Tel Aviv, Israel, Mar. 2000, pp. 775-784.

[5] Y. Gwon, R. Jain, and T. Kawahara, "Robust indoor location estimation of stationary and mobile users," in Proc. IEEE INFOCOM'04, Mar. 2004, pp. 1032-1043.

[6] K. Kaemarungsi and P. Krishnamurthy, "Modeling of indoor positioning systems based on location fingerprinting," in Proc. INFOCOM'04, Mar. 2004, pp. 1012-1022.

[7] Y. Chen and H. Kobayashi, "Signal strength based indoor geolocation," in Proc. IEEE ICC, 2002, pp. 436-439.

[8] M. Hossain, H. Nguyen Van, Y. Jin and W.-S. Soh, "Indoor localization using multiple wireless technologies," in Proc. IEEE MASS, Pisa, Italy, Oct. 2007, pp. 1-8. 